MS36-P22

\section{Using pressure to enhance the magnetic exchange interactions within $\mathrm{Cr}$ (III) dimers}

Helen Duncan ${ }^{1}$, Simon Parsons ${ }^{1}$, Euan Brechin ${ }^{1}$, Gopalan

Rajaraman ${ }^{2}$, Hector Fraser ${ }^{1}$

1. School of Chemistry, University of Edinburgh, Edinburgh, United Kingdom

2. Department of Chemistry, Indian Institute of Technology Bombay, Mumbai, India

email: h.duncan@ed.ac.uk

We have investigated a large series of $\mathrm{Cr}$ (III) dimer complexes with general formula $\left[\mathrm{Cr}_{2}(\mathrm{Me}-\mathrm{deaH})_{2}\left(\mathrm{O}_{2} \mathrm{CR}\right) \mathrm{Cl}_{2}\right]_{2}$. In these materials, magnetic properties are determined by highly deformable intermolecular interactions. The carboxylate above the $\mathrm{Cr} 2$ moiety distorts the planarity of the $\left[\mathrm{Cr}_{2} \mathrm{O}_{2}\right]$ unit, promoting a net ferromagnetic exchange between the metals.

Through substitution of $\mathrm{R}$ with eight organic groups, and DFT calculations the key structural contributions to the net exchange, which has both anti- and ferromagnetic contributions, have been identified. The key contributions are the out of plane angle, $\theta$, the $\mathrm{Cr}-\mathrm{O}-\mathrm{Cr}$ angle, $\varphi$, and the distortion of the planarity of the $\left[\mathrm{Cr}_{2} \mathrm{O}_{2}\right]$ unit, $\psi$ (Figure 1a, b, c respectively). [1]

We now use pressure to drive these structural transformations in three dimers where $\mathrm{R}=\mathrm{Me}$ (acetate), $\mathrm{tBu}$ (pivalate) and $\mathrm{Ph}$ (benzoate); a method which has previously successfully been used to enhance the magnetic exchange in Re(IV) molecular magnets. [2] Through applications of pressures up to $4.6 \mathrm{GPa}$, we have increased $\theta$ by up to 5.1(6) ${ }^{\circ}$ and $\psi$ by up to $1.4(2)^{\circ}$, (Figure $1 \mathrm{~d}$ ) which was predicted to increase the ferromagnetic contribution and decreased $\varphi$ by up to $2.0(4)^{\circ}$ which was predicted to increase the antiferromagnetic contribution.

Considering both the pressure-induced structural deformations, and the ambient pressure DFT we have predicted the optimum pressures to enhance the ferromagnetic exchange in these dimers, and compared the predictions against high-pressure magnetic susceptibility measurements. a)

c)
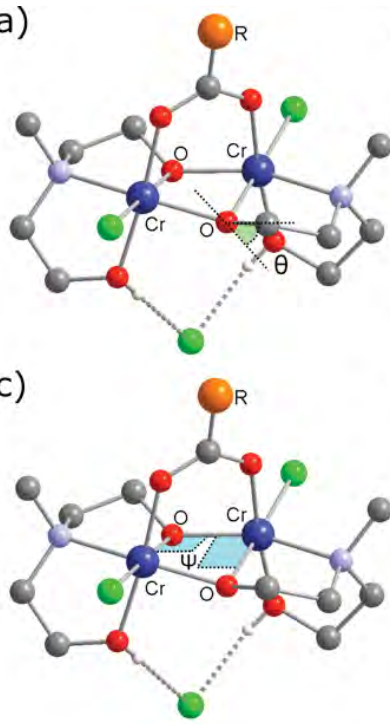

b)
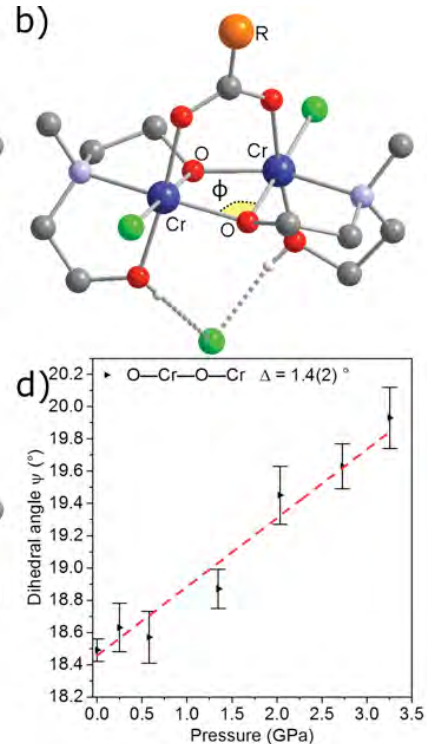

References:

[1] Fraser, H. W. L. et al. (2018). In preparation

[2] Woodall, C. H. et al. (2016). Nat. Commun, 7, doi:10.1038/ ncomms 13870

Keywords: High-pressure, Magnetism, Chromium 